\title{
A Three-Dimensional Numerical Model for Determining the Pressure Drops in Porous Media Consisting of Obstacles of Different Sizes
}

\author{
Y. Fumoto ${ }^{1}$, R. Liu ${ }^{2}$, Y. Sano ${ }^{*}, 1$ and X. Huang ${ }^{2}$ \\ ${ }^{I}$ Department of Mechanical Engineering, Shizuoka University, 3-5-1 Johoku, Naka-ku, Hamamatsu, 432-8561, Japan \\ ${ }^{2}$ School of Civil Engineering and Architecture, Wuhan Polytechnic University, 430023, P. R. China
}

\begin{abstract}
A three-dimensional numerical model is proposed to determine the pressure drops in porous media consisting of obstacles of different sizes. A series of full three-dimensional numerical calculations were performed to reveal complex three-dimensional velocity and pressure fields within three-dimensional porous structures consisting of spheres of different sizes. These numerical results are processed to obtain the macroscopic pressure gradients. An effective diameter concept has been proposed to correlate the resulting macroscopic pressure gradients with the Ergun equation. The most appropriate definition of the effective diameter has been found such that it, when substituted in the Ergun formula, gives the most reasonable estimate on the pressure drop for the given porosity and diameter distribution.
\end{abstract}

Keywords: Permeability, ergun equation, numerical simulation, pressure drop.

\section{INTRODUCTION}

The Ergun equation [1] is quite effective for estimating the pressure drops within packed beds of uniform particles. However, such an effective formula for the pressure drop estimation does not seem to exit when the bed consists of the particles of different sizes.

Liu et al., [2] proposed a formula for estimating an equivalent permeability of fractured porous media, assuming that the size of the particles embedded within the fractures is much smaller than the size of the fractures. Thus, they focused on the equivalent continuum model consisting of only two kinds of particles, whose sizes differ significantly from each other. The Brinkman-Darcy model was introduced to derive a rational mathematical model for determining the equivalent permeability of the fractured porous medium.

Sano et al., [3] extended their analytical expression to estimate the pressure drop in the flow in a bed packed with particles of different sizes. They conducted a series of the experiments using a column of particles of different sizes. Four sets of glass particles were mixed uniformly at a certain mixture ratio filling the vertical column to make various porous media consisting particles of different diameters. The values of effective permeability were obtained reading the slope of the measured water pressure-velocity plot. They concluded that the extended formula is valid for the ranges of the parameters studied. The parameter ranges studied by them, however, are rather limited, since they were able to construct the columns consisting of only four kinds of particles of different sizes. Furthermore, the complexity in their analytical expression makes its practical use difficult.

The pressure drop and its corresponding permeability can be determined by numerically applying the first principles of

*Address correspondence to this author at the Department of Mechanical Engineering, Shizuoka University, 3-5-1 Johoku, Naka-ku, Hamamatsu, 432-8561, Japan; Tel: +81-534-478-1046; Fax: +81-534-478-1046;

E-mail: f5945025@ipc.shizuoka.ac.jp mass and momentum conservation to flow of viscous fluids at the pore scale. Such theoretical attempts have been made by a number of investigators including Eidsath et al., [4], Couland et al., [5], Fowler and Bejan [6], Larson and Higdon [7], Nakayama et al., [8] and Nakayama and Kuwahara [9]. However, all these attempts were made for porous media consisting of uniform particles of the same size.

Nakayama et al., [10] recently conducted an exhaustive numerical experiment based on such first principles, using a unit structural model consisting of horizontal rods of different sizes. They proposed to use "effective diameter", which, when substituted in available empirical expressions, such as the Ergun formula [1], gives a reasonable estimate on the pressure drop in a porous medium consisting of obstacles of different sizes. After considerable computational manipulations, they concluded that the Ergun equation may be used even for evaluating the pressure drop of multi-sized obstacles. Their effective diameter of the multi-sized porous medium (to be substituted in the Ergun equation) is defined as follows:

$$
D_{e_{n}}=\frac{\int_{0}^{\infty} D^{n+1} h(D) d D}{\int_{0}^{\infty} D h(D) d D}
$$

where $h(D)$ is the density function for the distribution of diameter $D$. The exponent $n$ was varied from 0 to 2 to find best match between the numerical results and the Ergun equation. It was concluded that $n=1$ gives the best fit over wide ranges of the porosity and mixture ratio.

Since the effective diameter can easily be evaluated for the given diameter distribution, the concept of the effective diameter for multi-sized porous media is quite promising. Their conclusions however must be examined by carrying out full three-dimensional numerical calculations to eliminate the ambiguity resulting from the two-dimensionality of their unit structure model. 
In this study, three-dimensional numerical models of multi-sized structure are proposed to describe porous media consisting of particles of different sizes. Pore-scale numerical calculations are performed to find out the most appropriate definition of the effective diameter. Firstly, the microscopic numerical results are processed to determine the permeability for the given porosity. These values are examined to find out the appropriate definition of the effective diameter to be substituted in the Ergun formula. Secondly, the results are re-processed to evaluate the inertia effects, namely, the Forchheimer coefficient. Thus, the most appropriate definition of the effective diameter is sought, such that it, when substituted in the Ergun formula, gives the most reasonable estimate on the pressure drop for the given porosity and diameter distribution.

\section{THREE-DIMENSIONAL NUMERICAL MODELS}

Fig. (1) shows a structural unit of three-dimensional model, in which a body-centered cubic lattice of spheres and a face-centered cubic lattice of spheres are merged together to form a porous medium of two different sizes.

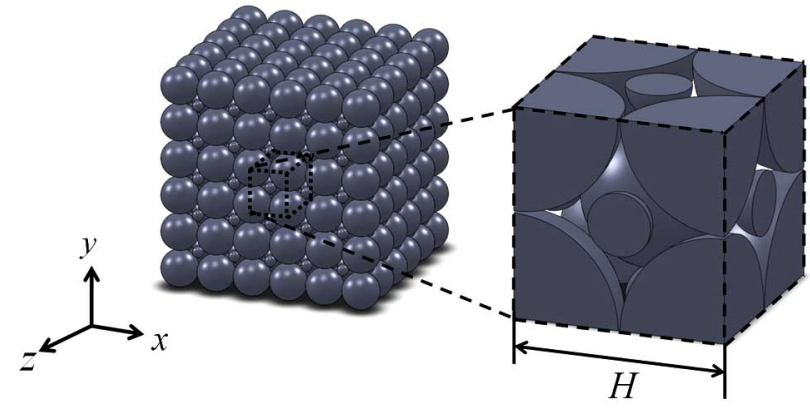

Fig. (1). A unit structure made of two kinds of spheres.

Table 1. Dimensions of the Unit Structure Made of Two Kinds of Spheres

\begin{tabular}{|c|c|c|c|c|}
\hline \multirow{2}{*}{ Model } & \multicolumn{3}{|c|}{ Particle diameter } & \multirow{2}{*}{ Porosity $\boldsymbol{\varepsilon}$} \\
\cline { 2 - 4 } & $\boldsymbol{D}_{\mathbf{1}} / \boldsymbol{H}$ & $\boldsymbol{D}_{\mathbf{2}} / \boldsymbol{H}$ & $\boldsymbol{D}_{\mathbf{3}} / \boldsymbol{H}$ & \\
\hline \hline 3-2a & 0.85 & 0.85 & 0.15 & 0.352 \\
\hline 3-2b & 0.3 & 0.3 & 0.7 & 0.433 \\
\hline 3-2c & 0.8 & 0.8 & 0.2 & 0.451 \\
\hline 3-2d & 0.2 & 0.2 & 0.7 & 0.453 \\
\hline 3-2e & 0.35 & 0.35 & 0.65 & 0.524 \\
\hline 3-2f & 0.20 & 0.20 & 0.65 & 0.560 \\
\hline 3-2g & 0.7 & 0.7 & 0.1 & 0.639 \\
\hline 3-2h & 0.65 & 0.65 & 0.35 & 0.645 \\
\hline 3-2i & 0.6 & 0.6 & 0.3 & 0.731 \\
\hline $3-2 \mathrm{j}$ & 0.4 & 0.4 & 0.5 & 0.732 \\
\hline
\end{tabular}

The sizes of particles forming the units and their porosities are listed in Table 1. Note that $D_{1}, D_{2}$ and $D_{3}$ are the diameter of the particles at the lattice corners, that in the centers and that at the face centers, respectively, whereas $H$ is the size of the structural unit. In the present cases of two different sizes, the diameter of the corner is set to the same as that in the center, $D_{1}=D_{2} \neq D_{3}$. The corresponding numerical models listed in the table are bird's-eye-viewed in Figs. (2a) to (2j) by labeling as $\mathbf{3 - 2} \mathbf{a}$ to $\mathbf{3 - 2} \mathbf{j}$, respectively.

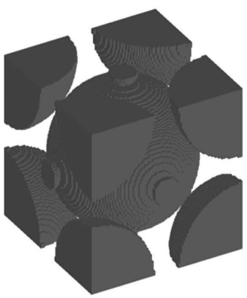

(a) $3-2 \mathrm{a}(\varepsilon=0.352)$

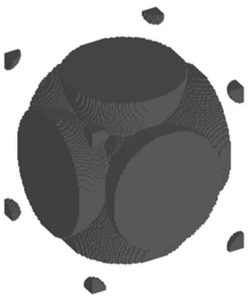

(d) $3-2 \mathrm{~d}(\varepsilon=0.453)$

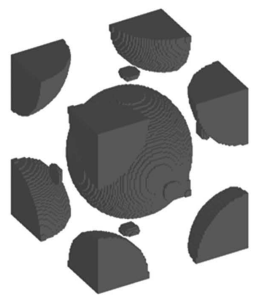

(g) $3-2 g(\varepsilon=0.639)$

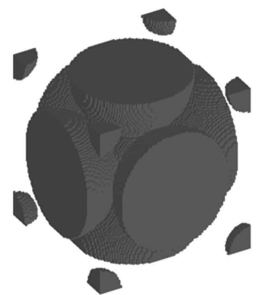

(b) $3-2 b(\varepsilon=0.433)$

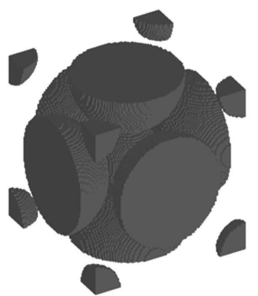

(e) $3-2 \mathrm{e}(\varepsilon=0.524)$

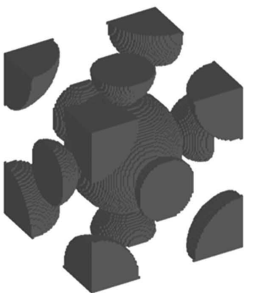

(h) $3-2 \mathrm{~h}(\varepsilon=0.645)$

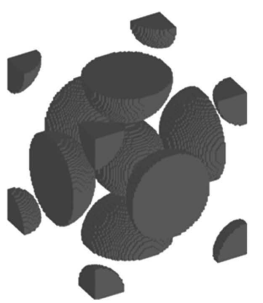

(j) $3-2 \mathrm{j}(\varepsilon=0.732)$

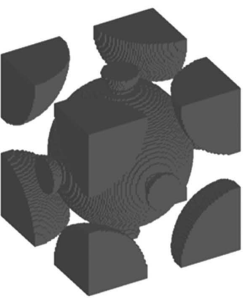

(c) $3-2 c(\varepsilon=0.451)$

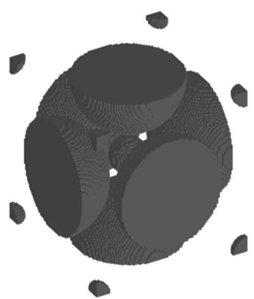

(f) $3-2 \mathrm{f}(\varepsilon=0.560)$

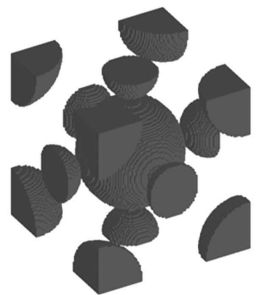

(i) $3-2 \mathrm{i}(\varepsilon=0.731)$
Fig. (2). Bird's-eye-views of the unit structures made of two kinds of spheres.

A three-dimensional numerical model for a porous medium of three different sizes is illustrated in Fig. (3), where a simple cubic lattice of spheres of $D_{1}$, a sphere of $D_{2}$ in the center and a face-centered lattice of spheres of $D_{3}$ are merged together to form a porous medium of three different sizes.

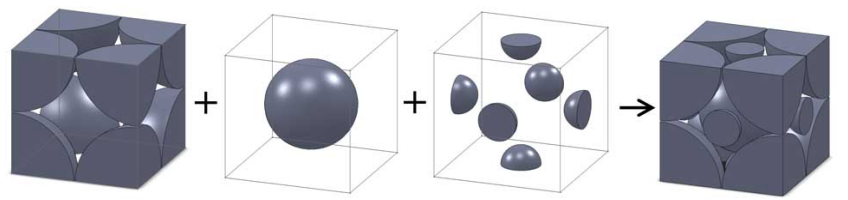

Fig. (3). A unit structure made of three kinds of spheres.

The dimensions of spheres forming the units and their porosities are listed in Table $\mathbf{2}$ for the cases of porous media of three kinds of spheres. The corresponding numerical models are bird's-eye-viewed in Figs. (4a) to (4h) by labeling as 3-3a to 3-3h, respectively. 
Table 2. Dimensions of the Unit Structure made of Three Kinds of Spheres

\begin{tabular}{|c|c|c|c|c|}
\hline \multirow{2}{*}{ Model } & \multicolumn{3}{|c|}{ Diameter } & \multirow{2}{*}{ Porosity $\mathcal{E}$} \\
\cline { 2 - 4 } & $\boldsymbol{D}_{\mathbf{1}} / \boldsymbol{H}$ & $\boldsymbol{D}_{\mathbf{2}} / \boldsymbol{H}$ & $\boldsymbol{D}_{\mathbf{3}} / \boldsymbol{H}$ & \\
\hline \hline 3-3a & 1 & 0.7 & 0.3 & 0.254 \\
\hline $3-3 \mathrm{~b}$ & 1 & 0.5 & 0.4 & 0.310 \\
\hline $3-3 \mathrm{c}$ & 0.8 & 0.4 & 0.6 & 0.359 \\
\hline $3-3 \mathrm{~d}$ & 0.95 & 0.65 & 0.25 & 0.383 \\
\hline $3-3 \mathrm{e}$ & 0.5 & 0.2 & 0.7 & 0.392 \\
\hline $3-3 \mathrm{f}$ & 0.2 & 0.3 & 0.7 & 0.443 \\
\hline $3-3 \mathrm{~g}$ & 0.9 & 0.6 & 0.1 & 0.504 \\
\hline $3-3 \mathrm{~h}$ & 0.6 & 0.8 & 0.2 & 0.617 \\
\hline
\end{tabular}

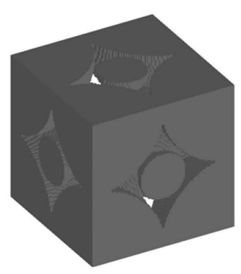

(a) $3-3 \mathrm{a}(\varepsilon=0.254)$

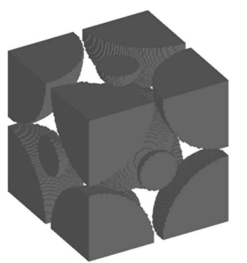

(d) $3-3 d(\varepsilon=0.383)$

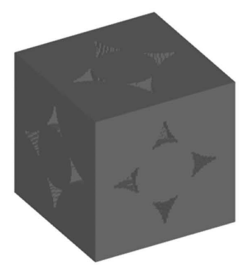

(b) $3-3 b(\varepsilon=0.310$

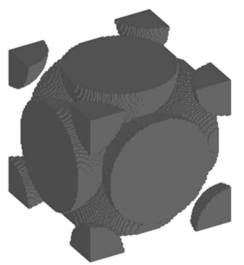

(e) $3-3 \mathrm{e}(\varepsilon=0.392)$

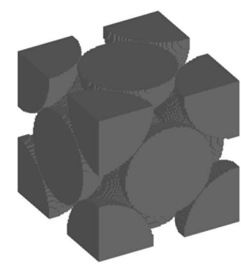

(c) $3-3 \mathrm{c}(\varepsilon=0.359)$

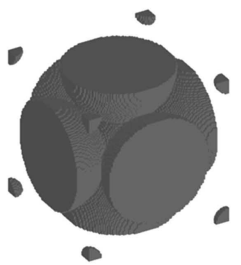

(f) $3-3 f(\varepsilon=0.443)$

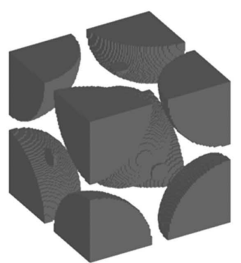

(g) $3-3 \mathrm{f}(\varepsilon=0.504)$

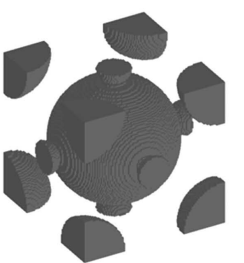

(h) $3-3 g(\varepsilon=0.617)$
Fig. (4). Bird's-eye-views of the unit structures made of three kinds of spheres.

\section{GOVERNING CONDITIONS \\ EQUATIONS AND \\ BOUNDARY}

A macroscopically uniform flow is assumed to prevail through an infinite number of the unit structures stacked together in a regular fashion. Only one structural unit of $H \times H \times H$ as shown in Fig. (5) can be taken as a calculation domain in consideration of the geometric periodicity. The direction of the macroscopically uniform flow is expressed in terms of $(\alpha, \beta)$ as illustrated in the figure such that

$\langle\vec{u}\rangle=|\langle\vec{u}\rangle|(\cos \alpha \cos \beta \vec{i}+\sin \alpha \vec{j}+\cos \alpha \sin \beta \vec{k})$ where $\langle\vec{u}\rangle$ denotes the Darcian velocity vector. The governing equations for the detailed flow field, namely, the equations of continuity and momentum, are given by,

$\nabla \cdot \vec{u}=0$

$(\vec{u} \cdot \nabla) \vec{u}=-\frac{1}{\rho} \nabla p+v \nabla^{2} \vec{u}$
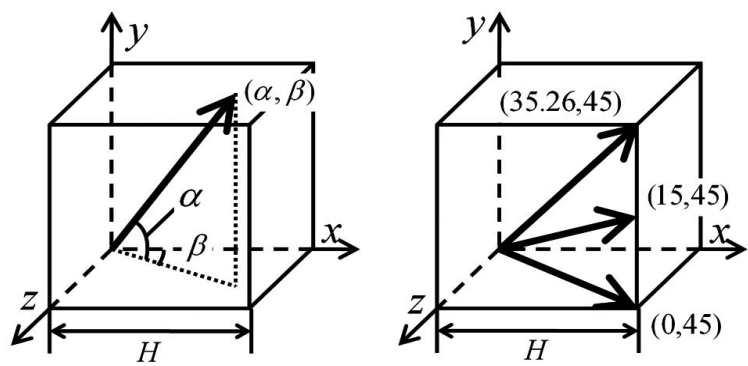

Fig. (5). Typical flow directions.

The periodic boundary and compatibility conditions are given by,

$$
\begin{aligned}
& \left.\vec{u}\right|_{x=0}=\left.\vec{u}\right|_{x=H} \\
& \left.\vec{u}\right|_{y=0}=\left.\vec{u}\right|_{y=H}
\end{aligned}
$$

$\left.\vec{u}\right|_{z=0}=\left.\vec{u}\right|_{z=H}$

$\left.\int_{0}^{H} \int_{0}^{H} u d y d z\right|_{x=0}=\left.\int_{0}^{H} \int_{0}^{H} u d y d z\right|_{x=H}$

$$
=H^{2}|\langle\vec{u}\rangle| \cos \alpha \cos \beta
$$

$\left.\int_{0}^{H} \int_{0}^{H} v d x d z\right|_{y=0}=\left.\int_{0}^{H} \int_{0}^{H} v d x d z\right|_{y=H}=H^{2}|\langle\vec{u}\rangle| \sin \alpha$

$$
\begin{array}{r}
\left.\int_{0}^{H} \int_{0}^{H} w d x d y\right|_{z=0}=\left.\int_{0}^{H} \int_{0}^{H} w d x d y\right|_{z=H} \\
=H^{2}|\langle\vec{u}\rangle| \cos \alpha \sin \beta
\end{array}
$$

Once the foregoing set of the governing equations are solved using the corresponding boundary and compatibility conditions, the macroscopic pressure gradient (i.e. the gradient of the intrinsic average pressure measured along the macroscopic flow direction) may readily be evaluated using the microscopic numerical results as follows:

$$
\begin{aligned}
-\frac{d\langle p\rangle^{f}}{d X}=\left\langle-\frac{\partial p}{\partial x}\right\rangle^{f} \cos \alpha \cos \beta+\left\langle-\frac{\partial p}{\partial y}\right\rangle^{f} \\
\\
\sin \alpha+\left\langle-\frac{\partial p}{\partial z}\right\rangle^{f} \cos \alpha \sin \beta
\end{aligned}
$$

where

$\left\langle-\frac{\partial p}{\partial x}\right\rangle^{f}=\frac{1}{A_{\mathrm{f}} H} \int_{A_{\mathrm{f}}}\left(\left.p\right|_{x=0}-\left.p\right|_{x=H}\right) d y d z$ 


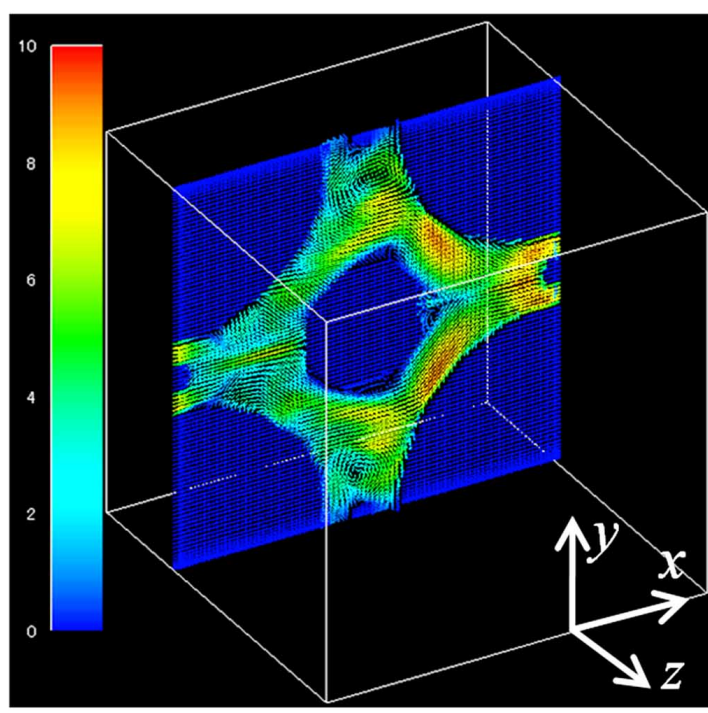

(a) $z / H=0.3,(\alpha, \beta)=(0,45)$

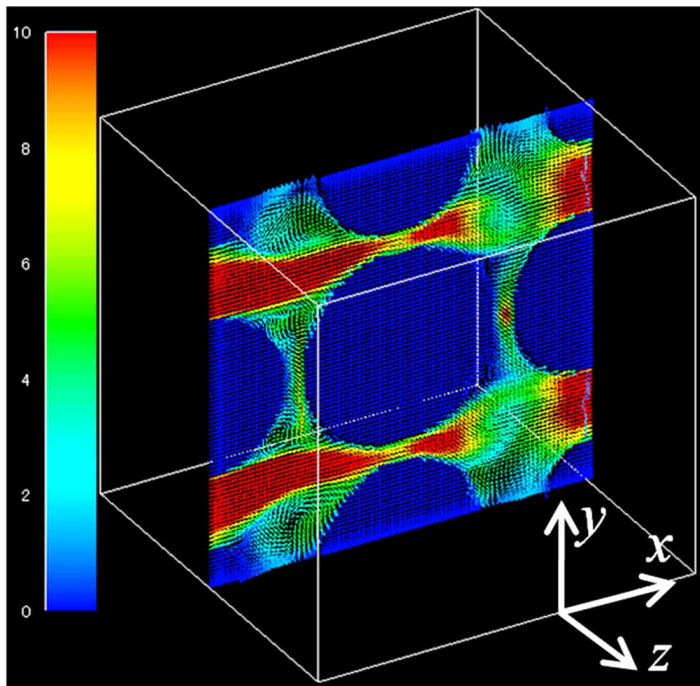

(c) $z / H=0.5,(\alpha, \beta)=(0,45)$

Fig. (6). Microscopic velocity fields in the model $3-3 \mathrm{~b}$ at $R e_{H}=300$.

$\left\langle-\frac{\partial p}{\partial y}\right\rangle^{f}=\frac{1}{A_{\mathrm{f}} H} \int_{A_{\mathrm{f}}}\left(\left.p\right|_{y=0}-\left.p\right|_{y=H}\right) d x d z$

$\left\langle-\frac{d p}{d z}\right\rangle^{f}=\frac{1}{A_{\mathrm{f}} H} \int_{A_{\mathrm{f}}}\left(\left.p\right|_{z=0}-\left.p\right|_{z=H}\right) d x d y$

where $A_{f}$ is the total area occupied by the fluid on the periodic boundary. The macroscopic pressure gradient depends on the macroscopic flow direction. In reality, a fluid particle travels through a porous medium, changing its direction freely. Thus, any numerical model has its limitations for simulating such random flow motions encountered in a real porous medium. For the first approximation, one may take an average of the macroscopic pressure gradients obtained for different macroscopic flow angles. A preli-

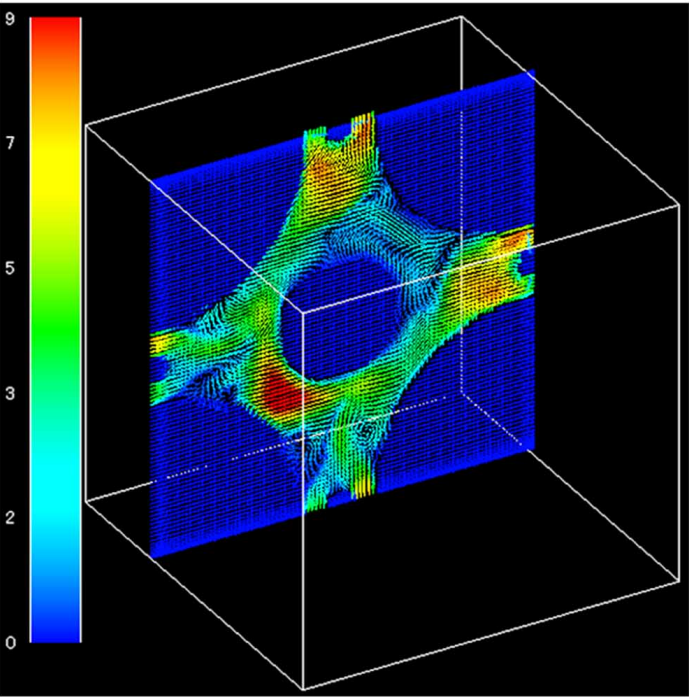

(b) $z / H=0.3,(\alpha, \beta)=(35.26,45)$

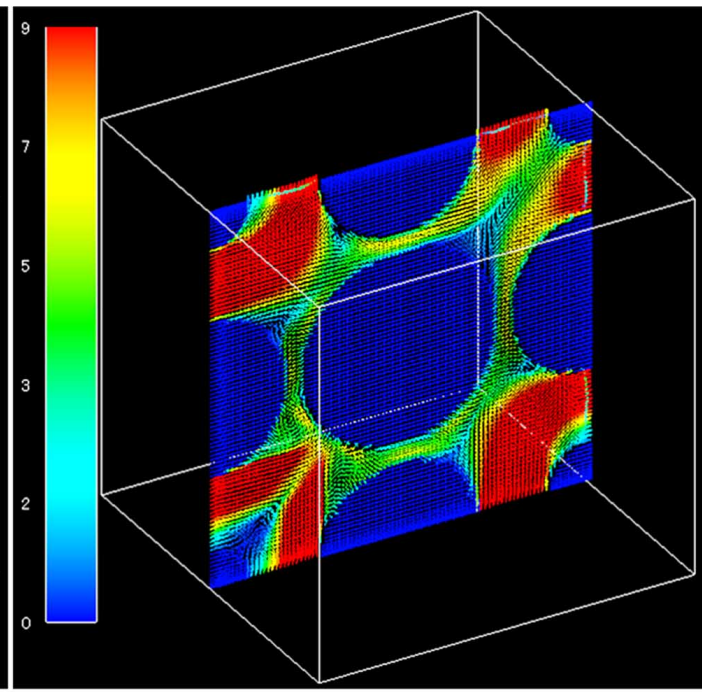

(d) $z / H=0.5,(\alpha, \beta)=(35.26,45)$ minary investigation on this respect revealed that the macroscopic pressure gradient obtained at a typical angle $(\alpha, \beta)=$ $\left(15^{0}, 45^{0}\right)$ is fairly close to its average over the flow angles. Thus, the results obtained at the flow angle $(\alpha, \beta)=\left(15^{0}\right.$, $45^{\circ}$ ) were used for determination of the permeability and inertia coefficient.

\section{METHOD OF COMPUTATION}

The foregoing governing equations are readily discretized by integrating them over a grid volume. SIMPLE algorithm for the pressure-velocity coupling, as proposed by Patankar and Spalding [11] were adopted to correct the pressure and velocity fields. Calculation starts with solving the two momentum equations, and subsequently, the estimated velocity field is corrected by solving the pressure 
correction equation reformulated from the discretized continuity and momentum equations, such that the velocity field fulfills the continuity principle. This iteration sequence must be repeated until convergence is achieved. Convergence can be measured in terms of the maximum change in each variable during an iteration. The maximum change allowed for the convergence check may be set to an arbitrarily small value (such as $10^{-5}$ ), as the variables are normalized by appropriate references. A fully implicit scheme was adopted with the hybrid differencing scheme for the advection terms. In order to check the grid dependency, the results obtained from a finer grid system $150 \times 150 \times 150$ are compared with those from the grid system $101 \times 101 \times 101$. After confirming the grid independency on the two sets of the solutions, calculations were carried out using only the grid system with $101 \times 101 \times 101$ nodes. Further details on this numerical procedure can be found in Patankar [12] and Nakayama [13].

\section{MICROSCOPIC VELOCITY FIELDS}

The resulting microscopic velocity fields are presented in Fig. (6) for the case of the unit made of three kinds of spheres, namely, the model labeled as 3-3b. The Reynolds number based on the unit length $H$ and the Darcian velocity was set to $\operatorname{Re}_{H}=|\langle\vec{u}\rangle| H / v=300$ such that the inertia effects on the velocity are appreciable. The velocity vectors are plotted on the cross-sectional planes at $z / H=0.3$ and 0.5 for the two cases of the flow angle, namely, $(\alpha, \beta)=\left(0^{\circ}, 45^{\circ}\right)$ and $\left(35.3^{0}, 45^{\circ}\right)$, which reveal the horizontally and diagonally symmetric velocity fields, respectively. Both sets of the figures clearly indicate the presence of three-dimensional bubbles in voids.

\section{MACROSCOPIC PRESSURE GRADIENT AND ITS CORRELATION}

According to the empirical Ergun equation, the macroscopic pressure gradient may be correlated as follows:

$$
-\frac{d\langle p\rangle^{f}}{d X}=\frac{\mu}{K}|\langle\vec{u}\rangle|+\rho b|\langle\vec{u}\rangle|^{2}
$$

where the permeability is given by

$$
K=\frac{\varepsilon^{3} D_{e}^{2}}{150(1-\varepsilon)^{3}}
$$

and the Forchheimer coefficient is given by

$$
b=1.75 \frac{1-\varepsilon}{\varepsilon^{3} D_{e}}
$$

The Ergun equation originally proposed for the packed beds of uniform spheres may now be extended for the cases of multi-scale structures made of different spheres by introducing the effective diameter $D_{e}$. In this study, the effective diameter $D_{e}$ is assumed to follow Equation (1), which, for the present three dimensional numerical models, reduces to
$D_{e_{n}}=\frac{D_{1}^{n+1}+D_{2}{ }^{n+1}+3 D_{3}^{n+1}}{D_{1}^{n}+D_{2}^{n}+3 D_{3}^{n}}$

Thus, one's task is to determine the exponent $n$ such that the Ergun equation (9) based on the effective diameter $D_{e_{n}}$ gives most reasonable estimate on the pressure gradient. $\mathrm{e}$ number of cases for the units made of two- and threedifferent sizes (as listed in Tables $\mathbf{1}$ and 2) were considered to generalize the outcome and to find out the most appropriate value for the exponent $n$.

As implied by the Ergun equation (9), the pressure gradient stays constant for small $R e_{H}$. Thus, following Nakayama et al. [8], the permeability $K$ can be obtained, by plotting the dimensionless permeability:

$\frac{K}{H^{2}}=\left.\frac{\mu|\langle\vec{u}\rangle|}{-\frac{d\langle p\rangle^{f}}{d X} H^{2}}\right|_{R e_{H} \rightarrow 0}=\left.\frac{1}{\frac{H^{2}}{K}+b H \operatorname{Re}_{H}}\right|_{R e_{H} \rightarrow 0}$

against the Reynolds number $R e_{H}$ and reading the value of the intercept as $R e_{H} \rightarrow 0$. One may refer to Nakayama and Kuwahara [9] for the details of the procedure. The Forchheimer coefficient, on the other hand, can be obtained, plotting the dimensionless Forchheimer coefficient:

$$
b H=-\left.\frac{d\langle p\rangle^{f}}{d X} \frac{H}{\rho|\langle\vec{u}\rangle|^{2}}\right|_{R e_{H} \rightarrow \infty}=\left.\left(\frac{H^{2}}{K} \frac{1}{R e_{H}}+b H\right)\right|_{R e_{H} \rightarrow \infty}
$$

against the Reynolds number $R e_{H}$, and reading its asymptotic value at $R e_{H} \rightarrow \infty$.

In order to seek the most appropriate exponent $n$, the permeability may be normalized using each $D_{e_{n}}$ (with $n=1$, 2 and 3) instead of $H$ as

$$
\frac{K}{D_{e_{n}}{ }^{2}}=\left(\frac{K}{H^{2}}\right) /\left(\frac{D_{e_{n}}}{H}\right)^{2}
$$

Similarly, the Forchheimer coefficient may be normalized using $D_{e_{n}}$ as,

$$
b D_{e_{n}}=(b H)\left(\frac{D_{e_{n}}}{H}\right)
$$

where

$$
\frac{D_{e 0}}{H}=\frac{D_{1}+D_{2}+3 D_{3}}{5 H}
$$

$$
\frac{D_{e_{1}}}{H}=\frac{D_{1}^{2}+D_{2}^{2}+3 D_{3}^{2}}{\left(D_{1}+D_{2}+3 D_{3}\right) H}
$$

$\frac{D_{e 2}}{H}=\frac{D_{1}^{3}+D_{2}^{3}+3 D_{3}^{3}}{\left(D_{1}^{2}+D_{2}^{2}+3 D_{3}^{2}\right) H}$ 
The numerical results obtained using various unit structures are processed to evaluate the macroscopic pressure gradients. The dimensionless values of the permeability, as defined Equation (15), are presented together in Figs. (7a), (7b) and (7c), in terms of $K / D_{e_{0}}{ }^{2}, K / D_{e_{1}}{ }^{2}$ and $K / D_{e_{2}}{ }^{2}$, respectively. The Forchheimer coefficients are also presented in Figs. (8a), (8b) and (8c), in terms of $b D_{e_{0}}, b D_{e_{1}}$ and $b D_{e_{2}}$, respectively.

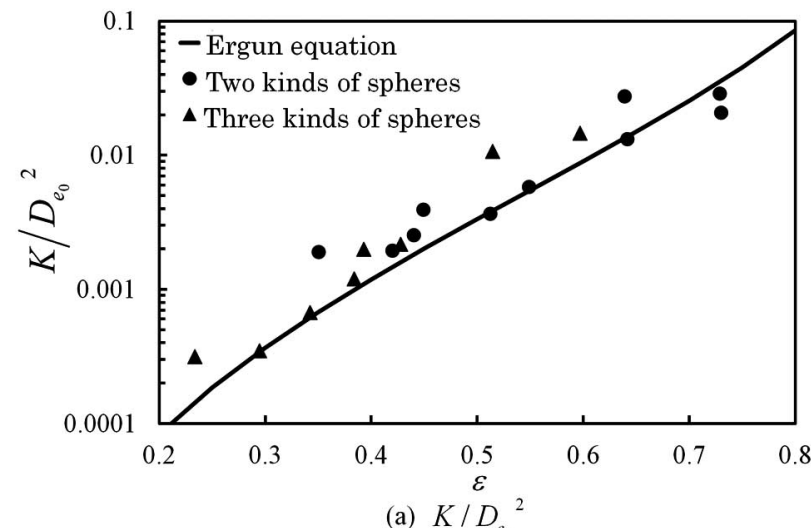

(a) $K / D_{e_{0}}{ }^{2}$

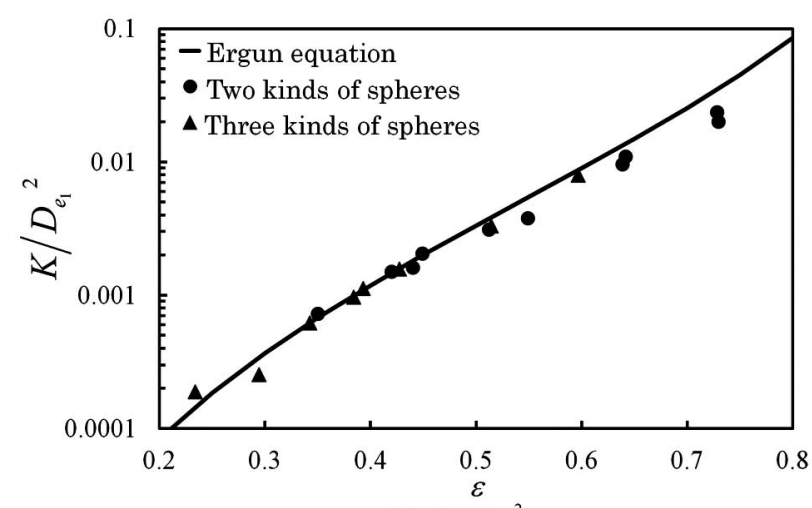

(b) $K / D_{e_{1}}{ }^{2}$

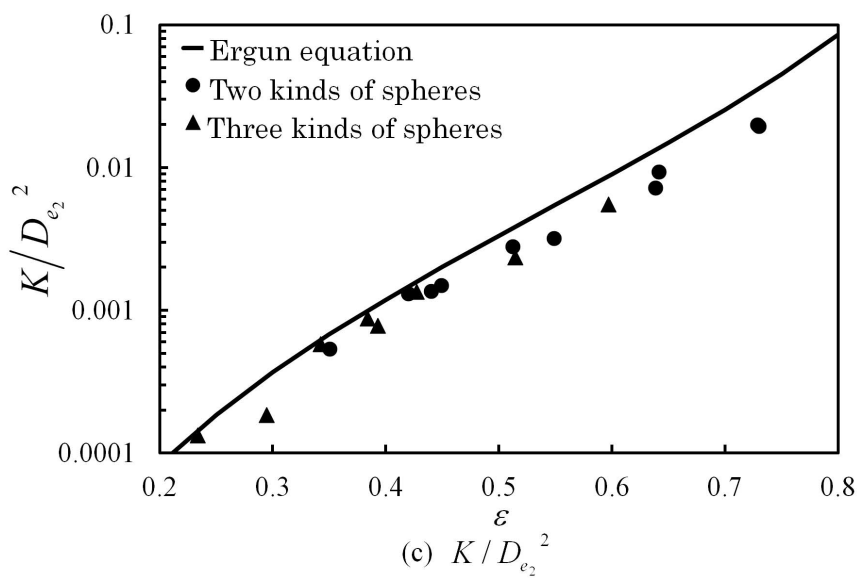

Fig. (7). Dimensionless permeability as a function of porosity (a) $K / D_{e_{0}}{ }^{2}$, (b) $K / D_{e_{1}}{ }^{2}$, (c) $K / D_{e_{2}}{ }^{2}$.

A comparison of Figs. (7a, b and c) reveals that $K / D_{e_{0}}{ }^{2}$ gives an estimate higher than the permeability of the Ergun equation $K / D_{e}{ }^{2}=\varepsilon^{3} / 150(1-\varepsilon)^{3}$, while $K / D_{e_{2}}{ }^{2}$ tends to underestimate it. Thus, $D_{e_{1}}$ appears to be the best choice for normalizing the permeability, as all data points in Fig. (7b) are clustered along the solid line of the Ergun equation.

As for the Forchheimer constant, $b D_{e_{0}}$ is not a good choice, since the data points of $b D_{e_{0}}$ in Fig. (8a) tend to depart from the Ergun equation as the porosity increases. As can be seen from Figs. $(\mathbf{8 b})$ and $(\mathbf{8 c})$, the data points of $b D_{e_{1}}$ and $b D_{e_{2}}$, on the other hand, are clustered along the Ergun

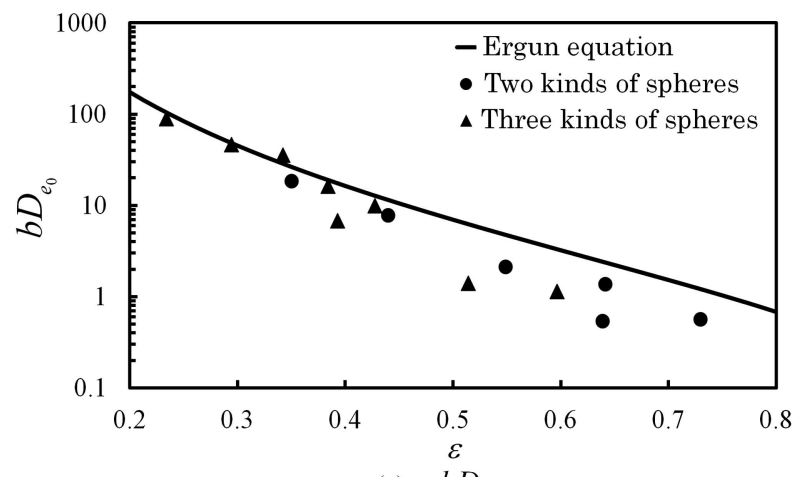

(a) $b D_{e_{0}}$

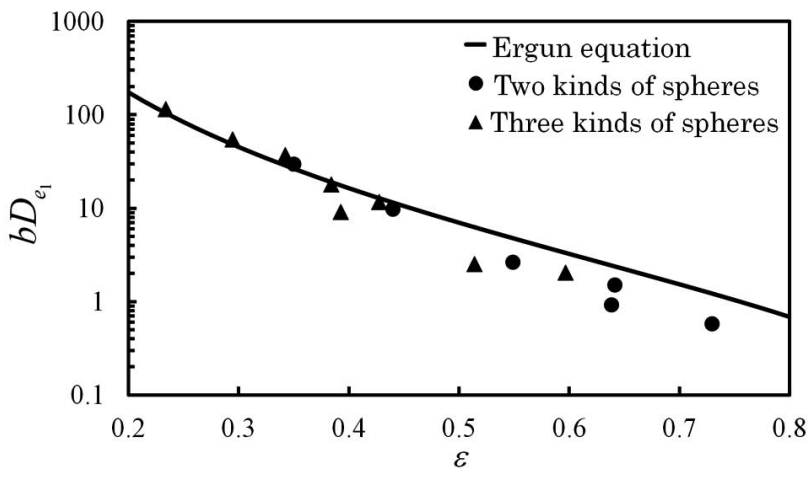

(b) $b D_{e_{1}}$

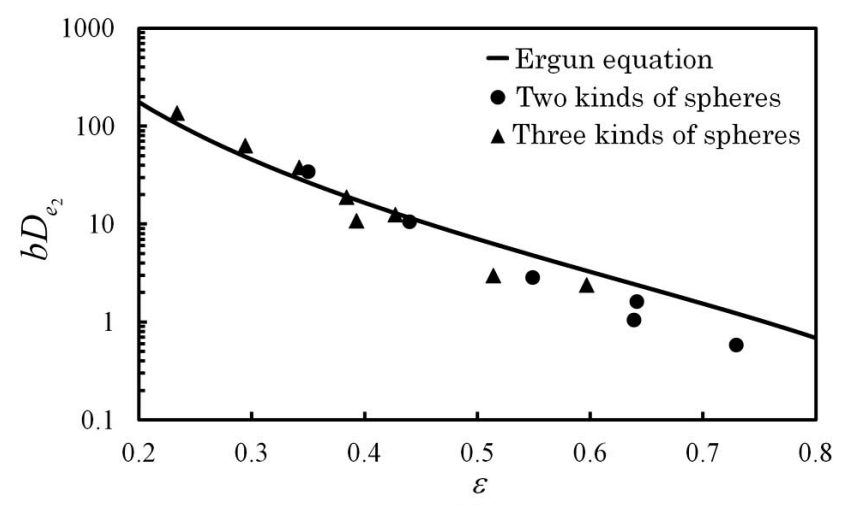

(c) $b D_{e_{2}}$

Fig. (8). Dimensionless Forchheimer constant as a function of porosity (a) $b D_{e_{0}}$, (b) $b D_{e_{1}}$, (c) $b D_{e_{2}}$. 
equation. In fact, it has been found that the inertia effects are rather insensitive to the way of normalization as long as the exponent is greater than or equal to 1 . Since $D_{e_{1}}$ is the best choice for the permeability, one may conclude that the most appropriate way of defining the effective average size of the multi-sized porous medium is given by Equation (1) with $n=1$, which, when substituted in the Ergun equation, gives a reasonable estimate on the pressure gradient. Hence, the following general formula can be used for porous media consisting of obstacles of different sizes:

$$
-\frac{d\langle p\rangle^{f}}{d X}=\frac{150(1-\varepsilon)^{2}}{\varepsilon^{3} D_{e_{1}}^{2}} \mu|\langle\vec{u}\rangle|+1.75 \frac{1-\varepsilon}{\varepsilon^{3} D_{e_{1}}} \rho|\langle\vec{u}\rangle|^{2}
$$

It is interesting to note that the conclusions drawn from this study based on the three-dimensional numerical models conform to those reported by Nakayama et al., [10] using the two-dimensional numerical models. The present threedimensional numerical models faithfully generate the empirical equation proposed by Ergun, even to the value of the Forchheimer coefficient, namely, 1.75, which was overestimated by Nakayama et al., [10] to be 2.5 in the twodimensional numerical models. Thus, the study reveals the importance of three-dimensionality in estimating the form drag within porous media.

\section{CONCLUSIONS}

Three-dimensional numerical models of multi-sized structure are proposed to describe porous media consisting of obstacles of different sizes. Pore-scale numerical calculations are performed to reveal complex three-dimensional velocity and pressure fields within three-dimensional porous structures consisting of spheres of different sizes. The most appropriate definition of the effective diameter was sought, such that it, when substituted in the Ergun formula, gives the most reasonable estimate on the pressure drop for the given porosity and diameter distribution. The present study reveals that the Ergun equation is quite effective for estimating the macroscopic pressure gradients even in porous media consisting of obstacles of different sizes, provided that the effective diameters are properly determined. In this study, only several kinds of simple structures have been investigated. A further computational investigation using more complex structures is required to draw a general conclusion on the specific expression of the effective diameter.

\section{NOMENCLATURE}

$$
\begin{array}{ll}
b & =\text { Forchheimer coefficient } \\
D_{l, 2,3} & =\text { Sphere diameters } \\
D_{e} & =\text { Effective diameter }(\mathrm{m}) \\
D_{e n} & =\text { Effective diameter with the exponent } n(\mathrm{~m}) \\
H & =\text { Size of structure }(\mathrm{m}) \\
K & =\text { Permeability }\left(\mathrm{m}^{2}\right)
\end{array}
$$

$$
\begin{array}{lll}
p & = & \text { Pressure } \\
\vec{u} & = & \text { Velocity vector }(\mathrm{m} / \mathrm{s}) \\
X & = & \text { Coordinate along the macroscopic flow } \\
& \text { direction } \\
x_{i} x, y, z= & \text { Cartesian coordinates }(\mathrm{m}) \\
\alpha, \beta \quad= & \text { Macroscopic flow angle (degree) } \\
\varepsilon & = & \text { Porosity }(-) \\
\mu & = & \text { Viscosity }(\mathrm{Pa} \mathrm{s}) \\
v & = & \text { Kinematic viscosity }\left(\mathrm{m}^{2} / \mathrm{s}\right) \\
\rho & = & \text { Fluid density }\left(\mathrm{kg} / \mathrm{m}^{2}\right)
\end{array}
$$

\section{SPECIAL SYMBOLS}

$$
\begin{aligned}
& \langle\rangle=\text { Darician average } \\
& \langle\rangle^{f}=\text { Intrinsic average }
\end{aligned}
$$

\section{ACKNOWLEDGEMENT}

None declared.

\section{CONFLICT OF INTEREST}

None declared.

\section{REFERENCES}

[1] S. Ergun, "Fluid flow through packed columns," Chem. Eng. Prog., vol. 48, pp. 89-94, 1952

[2] J.-J. Liu, Y. Sano, and A. Nakayama, "A simple mathematical model for determining the equivalent permeability of fractured porous media," Int. Comm., Heat Mass Transfer., vol. 30, pp. 220224, 2009.

[3] Y. Sano, K. Noguchi, and T. Kuroiwa, "An experimental investigation into the effective permiability of porous media whose matrices are composed of obstacles of different sizes," Open Transport Phenomena J., vol. 1, pp. 15-19, 2010.

[4] A. Eidsath, R.G. Carbonell, S. Whitaker, and L.R. Herman, "Dispersion in pulsed systems: comparison between theory and experiment for packed beds," Chem. Eng. Sci., vol. 38, pp. 1803$1816,1983$.

[5] O. Coulaud, P. Morel, and J.P. Caltagirone, "Numerical modeling of nonlinear effects in laminar flow through a porous medium," $J$. Fluid Mech., vol. 190, pp. 393-407, 1988.

[6] A.J. Fowler, and A. Bejan, "Forced convection in banks of inclined cylinders at low Reynolds numbers," Int. J. Heat Fluid Flow, vol. 15, pp. 90-99, 1994

[7] R.E. Larson, and J.J.L. Higdon, "A periodic grain consolidation model of porous media," Phys. Fluids, vol. A1, pp. 38-46, 1989.

[8] A. Nakayama, F. Kuwahara, Y. Kawamura, and H. Koyama, "Three-dimensional numerical simulation of flow through a microscopic porous structure," Proc. ASME/JSME Thermal Eng. Conf., vol. 3, pp. 313-318, 1995.

[9] A. Nakayama, and F. Kuwahara, "Numerical Modeling of Convective Heat Transfer in Porous Media Using Microscopic Structures" In: Handbook of Porous Media, K. Vafai, Ed. Marcel Dekker Inc.: NY 2000, pp. 441-488.

[10] A. Nakayama, R. Liu, Y. Sano, and X. Haung, "A General Formula for Determing the Permeability of Porous Media Consisting of Obstacles of Different Sizes," Phys. Numer. Simula. Geotech. Eng., vol. 1, pp. 3-8, 2010 
[11] S.V. Patankar, and D.B. Spalding, "A calculation procedure for heat, mass and momentum transfer in three-dimensional parabolic flows," Int. J. Heat Mass Transfer, vol. 15, pp. 1787-1806, 1972.
[12] S.V. Patankar, "Numerical heat transfer and fluid flow," Hemisphere Publishing Cooperation: Washington D.C., 1980.

[13] A. Nakayama, "PC-aided numerical heat transfer and convective flow," CRC Press: Boca Raton, 1995.

Received: June 13, 2011

Revised: October 10, 2011

Accepted: October 10, 2011

(C) Fumoto et al.; Licensee Bentham Open.

This is an open access article licensed under the terms of the Creative Commons Attribution Non-Commercial License (http:/creativecommons.org/ licenses/by-nc/3.0/), which permits unrestricted, non-commercial use, distribution and reproduction in any medium, provided the work is properly cited. 